\title{
Article \\ Aging Affects Multi-Objective Optimal Control Strategies during Obstacle Crossing
}

\author{
Chien-Chung Kuo ${ }^{1,2}{ }^{-}$, Jr-Yi Wang ${ }^{3,4}$, Sheng-Chang Chen ${ }^{3}$, Tung-Wu Lu ${ }^{3, *}$ and Horng-Chaung Hsu ${ }^{1,2}$ \\ 1 Department of Orthopedics, School of Medicine, China Medical University, Taichung 40433, Taiwan; \\ d4306@mail.cmuh.org.tw (C.-C.K.); d4749@mail.cmuh.org.tw (H.-C.H.) \\ 2 Department of Orthopedics, China Medical University Hospital, Taichung 40433, Taiwan \\ 3 Department of Biomedical Engineering, National Taiwan University, Taipei 10617, Taiwan; \\ 19036@s.tmu.edu.tw (J.-Y.W.); f90548028@gmail.com (S.-C.C.) \\ 4 Department of Orthopedics, Shuang Ho Hospital, Taipei Medical University, New Taipei 235041, Taiwan \\ * Correspondence: twlu@ntu.edu.tw
}

check for updates

Citation: Kuo, C.-C.; Wang, J.-Y.; Chen, S.-C.; Lu, T.-W.; Hsu, H.-C. Aging Affects Multi-Objective Optimal Control Strategies during Obstacle Crossing. Appl. Sci. 2021, 11, 8040. https://doi.org/10.3390/ app11178040

Academic Editor: Arkady Voloshin

Received: 1 August 2021

Accepted: 27 August 2021

Published: 30 August 2021

Publisher's Note: MDPI stays neutral with regard to jurisdictional claims in published maps and institutional affiliations.

Copyright: (c) 2021 by the authors. Licensee MDPI, Basel, Switzerland. This article is an open access article distributed under the terms and conditions of the Creative Commons Attribution (CC BY) license (https:// creativecommons.org/licenses/by/ $4.0 /)$.
Featured Application: Older adults adopt a crossing strategy that is different from that of the young, emphasizing foot-obstacle clearance to reduce the risk of tripping over the obstacle at the expense of increased energy expenditure. It appears that this strategy relies very much on the muscular strength of the lower extremities and the precision control of the end-points. Therefore, apart from other influencing factors, maintaining or improving muscle strength and the ability of limb position control is critical for safe and successful obstacle-crossing in the older population.

\begin{abstract}
Obstacle crossing challenges balance and increases the risk of falls in older people. Knowledge of the control strategies adopted by older people will be helpful for the study of the mechanisms of falls during obstacle crossing and the development of fall-prevention approaches. A mechanical model of the body combined with measured gait data was used to study the control strategies adopted by 17 healthy older and 17 young adults when crossing obstacles of different heights, in terms of the best-compromise weighting sets for the objectives of minimizing energy expenditure and maximizing the toe-obstacle and heel-obstacle clearances. The older group showed increased leading toe-obstacle clearance and trailing toe-obstacle distance, but decreased leading heel-obstacle distance. Compared with the young, the crossing strategy of older people emphasized the foot-obstacle clearance to reduce the risk of tripping, at the expense of energy expenditure. It appears that the multi-objective optimal control strategy relies on the muscular strength of the lower extremities and precise end-point control. Therefore, maintaining or improving the muscle strength and the ability of limb position control is critical for safe and successful obstacle-crossing in the older population.
\end{abstract}

Keywords: gait analysis; optimal control; obstacle crossing; aged

\section{Introduction}

Obstacle crossing challenges balance and is considered one of the daily activities that increases the risk of falls in older people [1-3]. Loss of balance and inability to recover balance are the main contributing factors to falling during obstacle crossing in older people [4]. For a successful obstacle crossing, necessary foot-clearance of the swing limb and stability of the body provided mainly by the stance limb are needed [5]. Insufficient footclearance will increase the risk of tripping, leading to loss of balance [6-8]. While increasing foot-clearance may help reduce the risk of tripping [9-11], the associated alteration of the whole-body posture may place greater challenges on body balance [6,7]. In this case, extra muscular effort and work at the joints in the stance limb will be required to maintain body balance or recover balance in cases where imbalance occurs. A previous study has reported the overall strategies for the simultaneous control of foot-clearance, lower limb muscular 
work, and total mechanical energy for successful obstacle crossing in young adults [12]. Similar knowledge in older adults will be helpful for developing intervention strategies for fall prevention in the older population.

Older people have been shown to cross obstacles with increased leading toe-clearance [13-15], slower crossing speed, shorter step length, and smaller step width [11], as well as increased muscular moments at the lower limb joints [9], when compared with young controls. Altered kinematics of individual joints of the lower extremities were also reported in previous studies $[5,9,10,13,16-18]$. While increased toe-clearance helps reduce the risk of tripping [9-11], it remains unclear how the reported changes in individual variables together would contribute to the higher risk of falls in older people. To gain insight into the control strategies of obstacle crossing, relative phase analysis has been used to study the patterns and variability of the multi-joint coordination during obstacle crossing in older people, which were independent of obstacle height $[15,19,20]$. While the mechanics of and coordination between individual joints have been studied, difficulties still exist in synthesizing these data to uncover the overall control of the locomotor system for obstacle crossing in older people. For example, older people changed some joint angles or moments with increasing obstacle height, while keeping other variables unchanged $[5,9,16]$. From these results, it is difficult to test whether there exists an obstacleheight independent control strategy present at the level of the central control system. To tackle this problem, Lu et al. proposed a multi-objective optimal control (MOOC) approach to study obstacle-crossing strategies in young adults from the perspective of the central control system [12].

Using the MOOC approach with a weighting method [21], the overall control strategy for obstacle crossing in young adults has been shown to be the best-compromise solution to an MOOC problem with conflicting objectives of maximizing foot clearance and minimizing mechanical energy expenditure. Minimizing energy expenditure has generally been accepted as the optimal control objective for normal walking [22-26], but not for obstacle crossing [9], even though both activities appear similar. During obstacle crossing, one has to lift the foot to cross the obstacle, so minimizing energy expenditure alone does not predict swing ankle trajectories that give the foot clearances observed in normal obstaclecrossing $[9,12]$. For a successful obstacle crossing, the swing foot has to cross over the obstacle with sufficient foot-clearance [5]. Previous studies have shown that older people crossed obstacles with increased toe-obstacle clearance, presumably to reduce the risk of tripping because, the greater the foot-obstacle clearance, the less likely the foot is to hit the obstacle [13]. Lifting the swing limb to increase the foot-obstacle clearance may increase the mechanical energy needed for the associated body postural adjustments while maintaining balance. However, no study has reported changes in energy expenditure and their possible relationship with the observed increase in toe-obstacle clearance during obstacle crossing in older people.

It has been shown in young adults that the weightings of the conflicting objectives of the MOOC problem for obstacle crossing were independent of obstacle height, suggesting that the control strategy was maintained in the central nervous system (CNS). Previous experimental studies have also indicated that, during obstacle crossing, a basic locomotor pattern was adjusted by higher CNS centers [27] rather than implementing a new pattern altogether $[18,28,29]$. With aging, it is well known that there is a natural degradation of the functions of the neuromusculoskeletal system, which is suspected of being responsible for the altered joint kinematic and kinetic changes during obstacle crossing. However, it remains unclear whether aging also affects the overall control strategy of obstacle crossing. Knowledge of the control strategies adopted by older people will be helpful for studying the mechanisms of falls during obstacle crossing and for developing prevention approaches.

The purpose of the current study was to use MOOC techniques with a model of the human body in the sagittal plane to study the control strategies adopted by healthy older and young people when crossing obstacles of different heights. The effects of age on the control strategies were investigated. It was hypothesized that, in order to reduce the risk of 
tripping over the obstacle, the older group would emphasize foot clearance with increased weightings - rather than energy expenditure - when compared with the young group.

\section{Materials and Methods}

\subsection{Subjects}

Seventeen healthy older adults (older group) and 17 BMI-matched healthy young adults (young group) participated in the current study (Table 1) with written informed consent as approved by the Institutional Review Board (IRB No. DMR98-IRB-072). They were all free of neuromusculoskeletal dysfunction and with normal or corrected vision. An a priori power analysis based on pilot results using $\mathrm{G}^{*}$ POWER [30] determined that a projected sample size of 11 subjects for each group would be needed for two-way mixeddesign analysis of variance (ANOVA) with a power of 0.8 and a large effect size (Cohen's $\mathrm{d}=0.8$ ) at a significance level of 0.05 . Thus, the current sample size for each group was adequate for the main objectives of the study.

Table 1. Means (standard deviations) of the basic characteristics of older and young participants.

\begin{tabular}{cccc}
\hline & Older & Young & $\begin{array}{c}\text { Group Effect } \\
(p \text {-Value })\end{array}$ \\
\hline Age, years & $72 \pm 6$ & $23 \pm 3$ & -- \\
\hline Gender, no. of females/males & $4 / 13$ & $4 / 13$ & -- \\
\hline BMI & $22.6 \pm 3.5$ & $22.2 \pm 2.1$ & 0.66 \\
\hline Height, $\mathrm{cm}$ & $160 \pm 6$ & $175 \pm 5$ & 0.01 \\
\hline Mass, $\mathrm{kg}$ & $58.7 \pm 8.5$ & $69.0 \pm 7.4$ & 0.01 \\
\hline
\end{tabular}

\subsection{Experimental Protocol}

In a university hospital gait laboratory, each subject walked at their preferred walking speed and crossed a tube-like obstacle placed across a height-adjustable frame [31]. The position and height of the obstacle were defined by two infrared-retroreflective markers placed on each end of the tube. The poses of the body segments were tracked using 39 infrared-retroreflective markers placed on specific anatomical landmarks commonly used in human motion analysis [32]. Three-dimensional trajectories of the markers were measured at $120 \mathrm{~Hz}$ using a seven-camera motion analysis system (Vicon 512, Oxford Metrics, London, UK), and the ground reaction forces (GRFs) and the center of pressure (COP) were measured at $1080 \mathrm{~Hz}$ using two forceplates (AMTI, Advanced Mechanical Technology, Watertown, MA, USA) placed on either side of the obstacle [33]. The test conditions were crossing obstacles of $10 \%, 20 \%$, and $30 \%$ of the subject's leg length, defined as the distance between the ipsilateral ASIS and medial malleolus, with each lower limb leading [34]. Data for three complete crossing trials for each lower limb leading were obtained for each obstacle height and each subject.

\subsection{Mathematical Model of the Body}

The human body was modeled as a sagittal seven-link system with rigid links of the upper body, the thighs, shanks, and feet connected by model hips, knees, and ankles as frictionless hinge joints (Figure 1) [12]. The model had seven degrees of freedom, corresponding to the seven angular displacements, namely, the ground / foot $\left(\theta_{T F}\right)$, ankle $\left(\theta_{T A}\right)$, knee $\left(\theta_{T K}\right)$, and hip $\left(\theta_{T H}\right)$ of the trailing stance limb, as well as the hip $\left(\theta_{L H}\right)$, knee $\left(\theta_{L K}\right)$, and ankle $\left(\theta_{L A}\right)$ of the leading swing limb (Figure 1$)$. The foot of the trailing stance limb was assumed to be connected to the ground at the ground/foot joint $\left(\theta_{T F}\right)$. The resultant forces and moments at the hinge joint were equivalent to the GRF. Model parameters, namely the lengths and inertial properties of the links and positions of joint centers, were obtained using a model-based optimization method that minimizes errors between model-predicted 
and measured COP positions during several calibration postures [31]. The governing equations of dynamics of the seven-link model are given as follows [12].

$$
M(q) \ddot{q}=T(t)+V(q) \dot{q}^{2}+G(q)+E(q, \dot{q})
$$

where $q$ is a $7 \times 1$ vector of the seven degree-of-freedom model variables, i.e., joint angular displacements; $\dot{q}$ and $\ddot{q}$ are joint angular velocities and accelerations, respectively; $M(q)$ is a $7 \times 7$ mass matrix; $T(t)$ is a $7 \times 1$ joint torque vector; $V(q) \dot{q}^{2}$ is a $7 \times 1$ vector of both Coriolis and centrifugal effects; $G(q)$ is a $7 \times 1$ gravitational force vector; and $E(q, \dot{q})$ is a $7 \times 1$ external force vector (Figure 1 ).

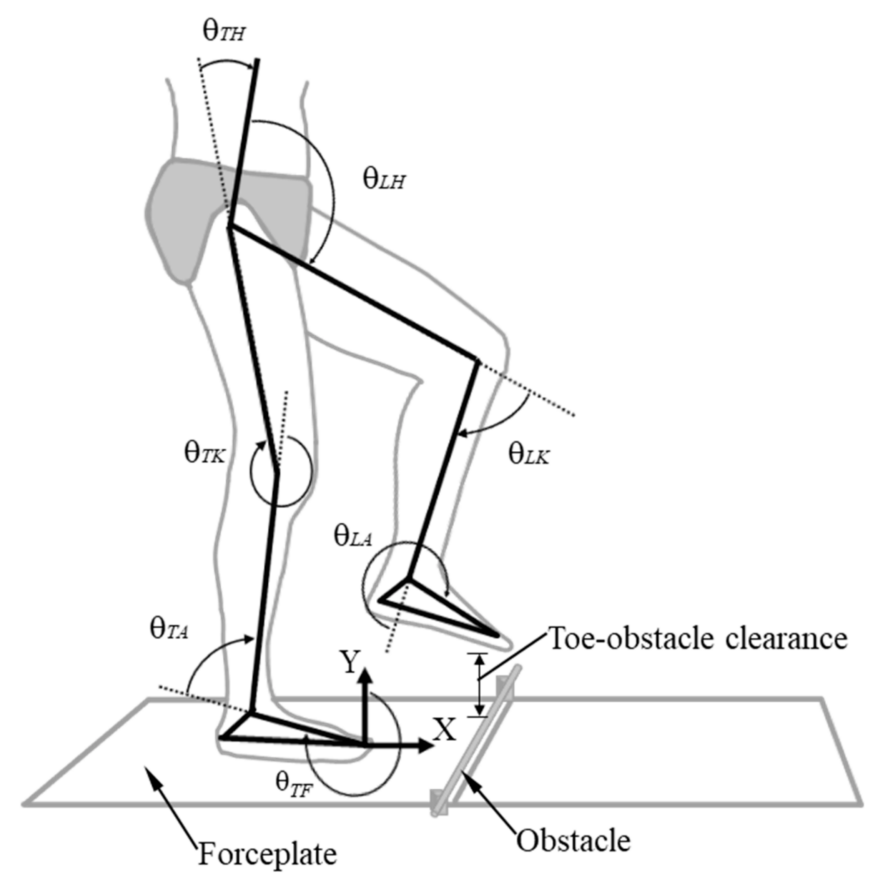

Figure 1. The sagittal seven-link model of the body during obstacle crossing with seven degrees of freedom, corresponding to the angular displacements: hip $\left(\theta_{L H}\right)$, knee $\left(\theta_{L K}\right)$, and ankle $\left(\theta_{L A}\right)$ of the leading swing limb, and foot $\left(\theta_{T F}\right)$, ankle $\left(\theta_{T A}\right)$, knee $\left(\theta_{T K}\right)$, and hip $\left(\theta_{T H}\right)$ of the trailing stance limb. Toe-obstacle clearance is also indicated, defined as the vertical distance of the toe above the obstacle. The $\mathrm{X}$-axis indicates the direction of progression.

\subsection{Multi-Objective Optimal Control (MOOC) of Obstacle Crossing}

For the current MOOC of obstacle crossing, some of the model variables were assigned with experimentally measured data, namely, the trailing foot $\left(\theta_{T F}\right)$, ankle $\left(\theta_{T A}\right)$, knee $\left(\theta_{T K}\right)$, and hip $\left(\theta_{T H}\right)$, and the leading ankle $\left(\theta_{L A}\right)$ (Figure 1). The measured swing ankle position $\left(y_{1}\right)$ at the toe-off immediately before crossing $\left(t_{1}\right)$, as well as the swing ankle position $\left(y_{n}\right)$ at the initial contact immediately after crossing $\left(t_{n}\right)$, were also taken as model input. Given these measured input data, the swing ankle trajectories would be determined by the MOOC laws. Considering the linkage of the leading swing limb, the angles of the leading hip $\left(\theta_{L H}\right)$ and knee $\left(\theta_{L K}\right)$ were determined once the positions of the swing hip and ankle were known (Figure 1). According to Lu et al., the MOOC of the lower extremities during obstacle crossing involves trade-offs between the conflicting objectives of minimizing mechanical energy and maximizing the swing heel and toe clearances [12]. Therefore, the MOOC problem of obstacle crossing in the current study was to search for the swing ankle trajectory, and thus the angular trajectories of the joints of the swing limb, and the torques of all the lower limb joints, that minimized the energy expenditure $\left(f_{1}\right)$ and maximized the heel-obstacle $\left(f_{2}\right)$ and toe-obstacle clearances $\left(f_{3}\right)$ above the obstacle. This MOOC problem was converted to a non-linear programming problem using a parameterization approach. 
The spatial trajectory of the swing ankle was discretized to $n$ positions over the total time of the swing phase, i.e., from toe-off $\left(t_{1}\right)$ to initial contact $\left(t_{n}\right)$ of the swing limb, at equal-timed intervals, $\Delta t=\frac{t_{n}-t_{1}}{n}$. The discretized swing ankle positions, i.e., $\bar{y}=\left(y_{1}, y_{2}, \ldots, y_{n}\right)$, were the design variables of the non-linear programming problem. The resulting parameterized MOOC problem can be described as follows:

Problem MOOC: Find a set of design variables, $\bar{y}=\left(y_{1}, y_{2}, \ldots, y_{n}\right)$ to minimize the following objective functions:

$$
\begin{gathered}
f_{1}(\bar{y})=\sum_{j=1}^{7} \sum_{i=1}^{n} T_{j}^{i} \omega_{j}^{i} \Delta t \\
f_{2}(\bar{y})=-d_{h} \\
f_{3}(\bar{y})=-d_{t}
\end{gathered}
$$

subject to the equations of dynamics of the seven-link model defined in Equation (1), where $f_{1}$ is the mechanical energy from the muscular works at the lower limb joints calculated as the sum of the products of the joint torques $\left(T_{j}^{i}\right)$ and joint angular velocities $\left(\omega_{j}^{i}\right)$ over all time intervals $(\Delta t)$ for all joints $(j), f_{2}$ is the negative of the toe-obstacle clearance $\left(d_{t}\right)$, and $f_{3}$ is the negative of the heel-obstacle clearance $\left(d_{h}\right)$ (Figure 1 ). Basically, the larger the value of $n$, the better the representation of the ankle trajectory. However, considering the dimensionality, and thus the computational effort, for solving the non-linear programming problem, a value of 20 was selected for $n$ in the current study [12].

The weighting method [21] was used to solve the MOOC problem by introducing a weighting factor to each objective function and converting the original problem into a new one with a single objective function as the weighted sum of the original objective functions, i.e., $\left(W_{1} f_{1}+W_{2} f_{2}+W_{3} f_{3}\right)$. $W_{1}, W_{2}$, and $W_{3}$ were the weighting factors for the energy expenditure $\left(f_{1}\right)$, heel-obstacle clearance $\left(f_{2}\right)$, and toe-obstacle clearance $\left(f_{3}\right)$, respectively, and satisfying $W_{1}+W_{2}+W_{3}=1$. For a set of prescribed weighting factors, $W_{1}, W_{2}$, and $W_{3}$, the converted MOOC problem was to find a set of design variables, $\bar{y}=\left(y_{1}, y_{2}, \ldots, y_{n}\right)$, to minimize the following objective function:

$$
f(\bar{y})=W_{1} \frac{f_{1}(\bar{y})}{f_{1}^{*}}+W_{2} \frac{f_{2}(\bar{y})}{f_{2}^{*}}+W_{3} \frac{f_{3}(\bar{y})}{f_{3}^{*}}
$$

where $f_{1}^{*}, f_{2}^{*}$, and $f_{3}^{*}$ were the optimum objective function values of the non-linear programming problem with each objective function as the single objective. Each objective function was normalized by its single-objective optimum value to be non-dimensionless so that the objective functions became mensurable. For a given set of values for the design variables, inverse dynamics analysis was performed with the seven-link model to obtain the necessary quantities for the solution of the MOOC problem, including joint angles and torques of the lower limbs. The best-compromise solution to the MOOC problem was the optimum solution to the new problem defined in Equation (5) with a set of weighting factors $\left(W_{1}, W_{2}, W_{3}\right)$ that produced an ankle trajectory that best matched the measured trajectory, giving the minimum root mean squared errors (RMSEs). In the current study, the MOOC-associated problems were solved using in-house software developed in MATLAB (Mathworks, Boston, MA, USA).

To obtain the experimental input data for solving the MOOC problem, a validated 3D model of the locomotor system [35] was used to calculate the joint angles and moments from experimental data via inverse dynamics analysis and then projected onto the sagittal plane. The RMSEs between the MOOC model-predicted and experimentally measured leading ankle trajectories, as well as joint angles of the leading limb and joint moments of the trailing stance limb, were calculated for all height conditions and each subject. 


\subsection{Statistical Analysis}

A two-way mixed-design analysis of variance (ANOVA) with one between-subject factor (group) and one within-subject factor (obstacle height) was used to compare the best-compromise weighting sets, energy expenditure $\left(f_{1}\right)$, end-point control variables, and RMSEs of the calculated model variables, including the ankle positions and joint angles and moments. If an interaction was found, pair-wise between-group comparisons were performed using an independent t-test for each obstacle height, and a post hoc trend analysis was performed to determine the trend of the variable with increasing obstacle height for each group. If there was no interaction, the main effects were reported. All significance levels were set at $\alpha=0.05$. SPSS version 20 (SPSS Inc., Chicago, IL, USA) was used for all statistical analyses.

\section{Results}

There were no statistical interactions between group and obstacle height factors for any tested variables, so only the main effects are reported here. The older group was found to have significantly greater leading toe-clearance, energy expenditure, and longer trailing toe-obstacle distances, but shorter leading heel-obstacle distances (Table 2 and Figure 2). With increasing obstacle height, the energy expenditure was significantly increased linearly (Table 2).

Table 2. Means (standard deviations) of the end-point variables and energy expenditure for the older and young control groups when crossing obstacles of three different heights.

\begin{tabular}{|c|c|c|c|c|c|}
\hline & \multicolumn{3}{|c|}{ Obstacle Height (\% Leg Length) } & Group Effect & Height Effect \\
\hline & 10 & 20 & 30 & \multicolumn{2}{|c|}{$p$-Value } \\
\hline \multicolumn{6}{|c|}{ Leading heel-obstacle distance (\% Leg length) } \\
\hline Older & $17.8(4.9)$ & $17.3(3.5)$ & $16.9(3.9)$ & \multirow{2}{*}{$0.01 *$} & \multirow{2}{*}{0.23} \\
\hline Young & $20.3(2.6)$ & $20.2(2.4)$ & $19.5(2.2)$ & & \\
\hline \multicolumn{6}{|c|}{ Trailing toe-obstacle distance (\% Leg length) } \\
\hline Older & $24.9(4.4)$ & $24.6(4.2)$ & $24.2(4.8)$ & \multirow{2}{*}{$0.01 *$} & \multirow{2}{*}{0.18} \\
\hline Young & $21.0(2.7)$ & $20.4(3.1)$ & $20.6(2.9)$ & & \\
\hline \multicolumn{6}{|c|}{ Leading-toe clearance (mm) } \\
\hline Older & $156(34)$ & $169(33)$ & $176(40)$ & \multirow{2}{*}{$0.04 *$} & \multirow{2}{*}{0.57} \\
\hline Young & 149 (25) & $144(26)$ & $128(20)$ & & \\
\hline \multicolumn{6}{|c|}{ Leading-heel clearance $(\mathrm{mm})$} \\
\hline Older & $130(31)$ & $137(34)$ & $142(39)$ & \multirow{2}{*}{0.96} & \multirow{2}{*}{0.24} \\
\hline Young & $135(27)$ & $137(35)$ & 135 (33) & & \\
\hline \multicolumn{6}{|c|}{ Energy expenditure $(\%$ body weight $\times$ leg length $\times$ total time) } \\
\hline Older & 174.4 (41.7) & $172.8(30.1)$ & $183.2(35.9)$ & \multirow{2}{*}{$0.03 *$} & \multirow{2}{*}{$0.03 \uparrow$} \\
\hline Young & $149.3(22.6)$ & $145.4(20.8)$ & $156.3(19.3)$ & & \\
\hline
\end{tabular}

*: significant difference between groups. $\uparrow$ : significant linearly increasing trend. 

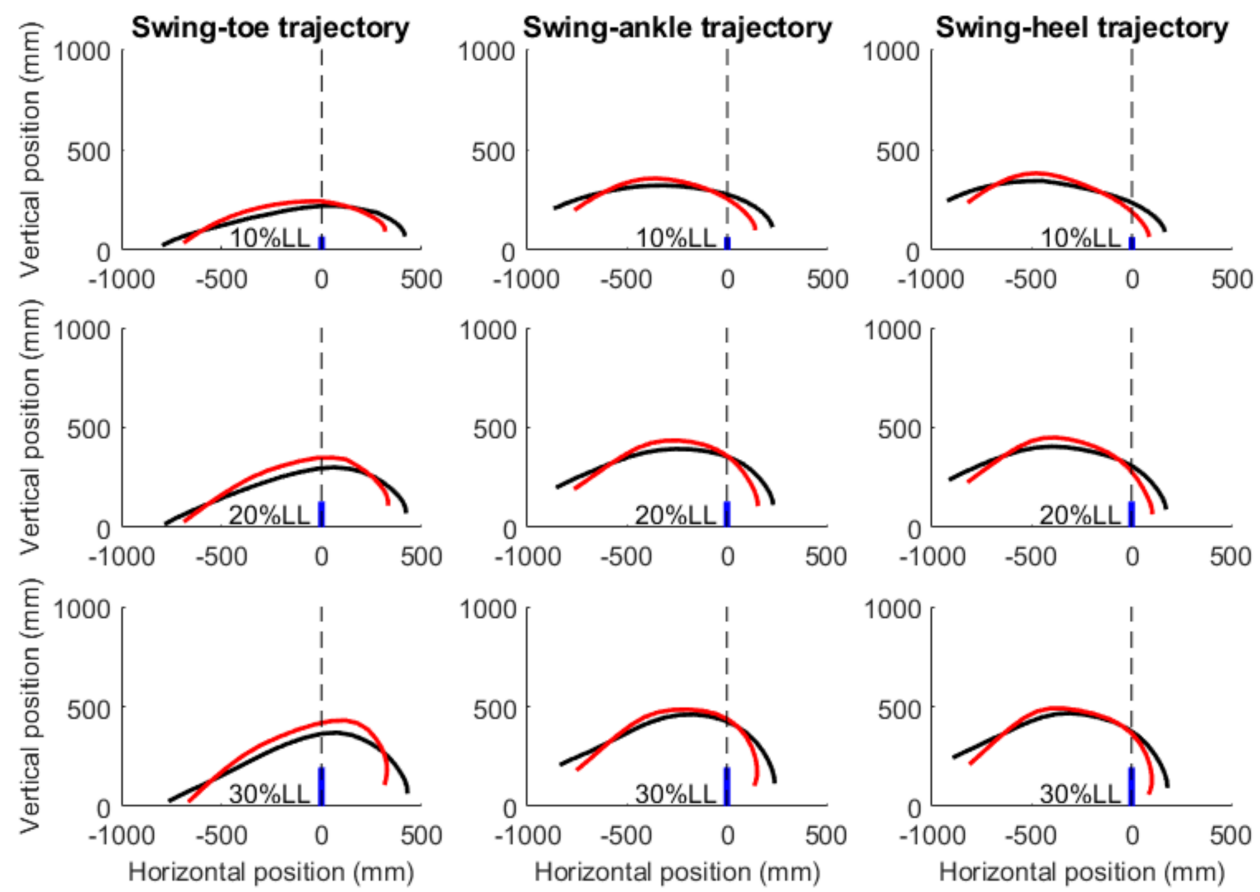

Figure 2. Sagittal trajectories of the ankle, toe, and heel of the leading swing limb of typical subjects of the older (red curves) and young (black curves) groups when crossing obstacles (solid blue lines) of $10 \%, 20 \%$, and $30 \%$ of leg length (LL).

The older group showed significantly increased weightings to the heel- and toeclearances, but decreased energy expenditure weightings (W1: 0.533; W2: 0.233; W3: 0.233) when compared with the young group (W1: 0.68; W2: 0.16; W3: 0.16) $(p<0.01$, Figure 3). There were no significant height effects for the best-compromise weightings $(p>0.05$, Figure 3).

$\begin{array}{ccc}\text { Energy expenditure } & \text { Heel-clearance } & \text { Toe-clearance } \\ \text { (W1) } & \text { (W2) } & \text { (W3) }\end{array}$

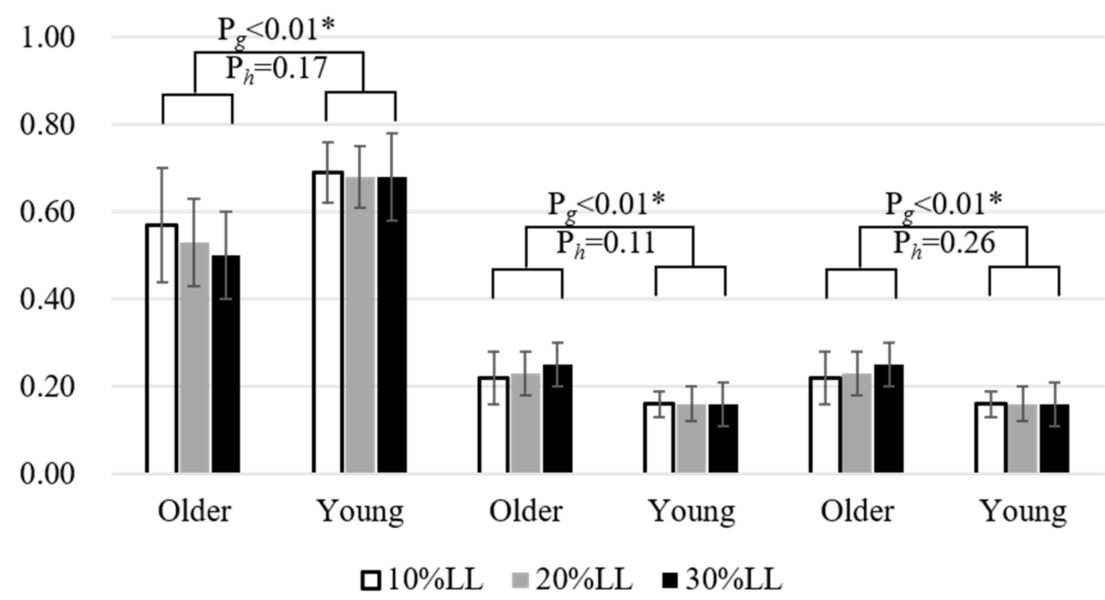

Figure 3. Means (standard deviations) of the best-compromise weighting sets (W1, W2, W3) for the older and young groups when crossing obstacles of three different heights: $10 \%$ (white), 20\% (grey), and 30\% (black) leg length (LL). $p$-values for the main effects are also given (Pg: group effects; Ph: height effects). Asterisks indicate statistically significant differences $(p<0.05)$.

With the best-compromise weighting sets, the RMSEs of the leading ankle trajectories over the swing phase between MOOC model predictions and experimental measurements 
were less than $8 \mathrm{~mm}$ for all test conditions, without significant group or height effects $(p>0.05$, Table 3). Similarly, the RMSEs for the swing hip and knee angles were less than $2^{\circ}$ for all test conditions, without significant group or height effects (Table 3). The RMSEs for the joint moments of the trailing stance limb were less than about $5 \mathrm{Nm}$ for both groups and all obstacle conditions, without significant group or height effects (Table 3 ).

Table 3. Means (standard deviations) of the RMSEs of the kinematic data of the leading swing limb and the trailing joint moments between the best-compromise solutions of the Multi-Objective Optimal Control (MOOC) and the experimental data.

\begin{tabular}{|c|c|c|c|c|c|}
\hline & \multicolumn{3}{|c|}{ Obstacle Height (\% Leg Length) } & \multirow{2}{*}{$\begin{array}{r}\text { Group Effect } \\
p-\end{array}$} & \multirow{2}{*}{$\begin{array}{l}\text { Height Effec } \\
\text { lue }\end{array}$} \\
\hline & 10 & 20 & 30 & & \\
\hline \multicolumn{6}{|c|}{ Ankle trajectory (mm) } \\
\hline Older & $7.1(3.1)$ & $7.1(2.9)$ & $7.8(1.7)$ & \multirow{2}{*}{0.4} & \multirow{2}{*}{0.63} \\
\hline Young & $6.7(1.9)$ & $6.7(2.3)$ & $7.3(2.3)$ & & \\
\hline \multicolumn{6}{|c|}{ Swing hip angle $\left(^{\circ}\right)$} \\
\hline Older & $0.84(0.22)$ & $0.81(0.36)$ & $0.98(0.29)$ & \multirow{2}{*}{0.11} & \multirow{2}{*}{0.11} \\
\hline Young & $0.64(0.19)$ & $0.66(0.28)$ & $0.74(0.23)$ & & \\
\hline \multicolumn{6}{|c|}{ Swing knee angle $\left(^{\circ}\right)$} \\
\hline Older & $1.71(0.49)$ & $1.39(0.62)$ & $1.40(0.33)$ & \multirow{2}{*}{0.08} & \multirow{2}{*}{0.09} \\
\hline Young & $1.41(0.39)$ & $1.29(0.43)$ & $1.26(0.38)$ & & \\
\hline \multicolumn{6}{|c|}{ Trailing ankle moment (Nm) } \\
\hline Older & $3.13(0.72)$ & $3.77(1.64)$ & $3.90(0.81)$ & \multirow{2}{*}{0.73} & \multirow{2}{*}{0.11} \\
\hline Young & $3.44(0.76)$ & $3.71(1.18)$ & $4.00(0.97)$ & & \\
\hline \multicolumn{6}{|c|}{ Trailing knee moment (Nm) } \\
\hline Older & $3.08(0.84)$ & 3.05 (1.68) & $2.99(0.80)$ & \multirow{2}{*}{0.59} & \multirow{2}{*}{0.37} \\
\hline Young & $2.75(0.50)$ & $3.39(0.85)$ & $3.53(0.88)$ & & \\
\hline \multicolumn{6}{|c|}{ Trailing hip moment (Nm) } \\
\hline Older & $3.63(0.90)$ & $4.02(1.96)$ & $3.98(1.11)$ & \multirow{2}{*}{0.11} & \multirow{2}{*}{0.68} \\
\hline Young & $4.35(0.74)$ & $4.59(1.08)$ & $4.46(1.02)$ & & \\
\hline
\end{tabular}

\section{Discussion}

The current study aimed to use multi-objective optimal control techniques with a mechanical model of the human body in the sagittal plane to study the control strategies adopted by healthy young and older adults when crossing obstacles of different heights. The older group was found to cross obstacles with significantly increased weightings to the heel- and toe-clearances, but decreased energy expenditure weightings when compared with the young group (Figure 3), supporting the hypothesis posed in the current study. These best-compromise weighting sets were not affected by obstacle height, suggesting that the observed strategy was one at the central nervous system level. The current findings suggest that the older adults adopted a crossing strategy that is different from that of the young, emphasizing the foot clearance to reduce the risk of tripping over the obstacle at the expense of increased energy expenditure.

The older subjects crossed obstacles with altered end-point control when compared with the young, as indicated by the increased leading toe-obstacle clearance and trailing toe-obstacle distance, but decreased leading heel-obstacle distance. Similar age effects on toe-obstacle clearances were also found in the literature [13,15,36-38]. Increased leading toe-obstacle clearance appears to be helpful for reducing the risk of tripping during obstacle crossing in older people $[13,15]$. For an increased leading toe-obstacle clearance, an efficient approach would be to cross the obstacle with the trailing stance foot closer to the obstacle [37]. However, the older subjects were found to cross with increased trailing toe-obstacle distance, but with subsequently reduced heel-obstacle distance when com- 
pared with the young (Table 2). Reduced heel-obstacle distance may increase the risk of stumbling, although heel or midsole contact has been suggested to carry less risk for a fall than toe contact [11]. On the other hand, increased trailing toe-obstacle distance may increase the separation of the body's center of mass relative to the center of pressure, increasing the risk of loss of balance [39].

The observed obstacle-height-independent end-point trajectories for obstacle crossing were a result of the best compromise between minimizing mechanical energy and maximizing the toe-obstacle and heel-obstacle clearances. However, the strategy was multi-objective and specific to different age groups, which was in contrast to the singleobjective and possibly age-independent control strategy for unobstructed level walking (i.e., minimization of energy expenditure, [22]). Note that the best-compromise weighting sets in both groups were not affected by obstacle height, suggesting that the control strategies for obstacle crossing were stored and executed at the central nervous system level [12] according to the central pattern generator theory [40]. This best-compromise MOOC strategy was found to change with aging. The current results indicate that the older adults placed more emphasis on foot-obstacle clearances than on energy expenditure compared with the healthy young group. The significantly increased weightings for the foot-obstacle clearance corresponded to the increased values of the toe-obstacle clearances, irrespective of obstacle heights, suggesting a strategy for reduced risk of tripping during obstacle crossing. However, this comes with the expense of increased mechanical energy expenditure (decreased weighting for minimization of mechanical energy) owing to the conflicting nature of the objectives for obstacle crossing (Table 2). Increased mechanical energy expenditure indicated increased muscular work at the lower limb joints was needed for the stability of the stance limb (Equation (2)) when the swing limb was lifted to increase toe-obstacle clearance. This is a challenge to older people who often have reduced muscle strength during the aging process [41-43]. It has been noted that obstacle negotiation is a precise end-point control task $[9,13,18,44,45]$. Sufficient foot-obstacle clearance and necessary body stability provided mainly by the stance limb are essential to a successful and safe obstacle crossing [13]. It appears that the MOOC strategy adopted by the older group would rely on not only the muscular strength of the lower extremities for stance stability, but also the ability of precision end-point control for the increased toe-obstacle clearance. Both the ability of end-point control and muscle strength are important components for preventing falls in older people [46-49]. Fall risks may be increased if an older person has muscle weakness [41-43] or compromised limb position control [11,41,50], or increased variability in performing motor tasks [15,51,52], which are common in the process of aging. Therefore, apart from factors such as vision, spatial cognition, and fear of tumbling, maintaining or improving muscle strength and the ability of limb position control are critical for safe and successful obstacle crossing in the older population.

The current study approached the complicated control problem of obstacle crossing with the multi-objective optimal control technique considering the laws of kinematics and kinetics. The small RMSEs of the ankle trajectories, leading joint angles, and trailing joint moments indicate that the iterative solution process converged and that the bestcompromise solutions for the MOOC problems were obtained with a high degree of accuracy. This enabled the study of the effects of aging on the control strategies of obstacle crossing via a reliable comparison of the best-compromise weighting sets between the older and young groups. In the current study, the crossing strategies over the swing phase of the leading limb during obstacle crossing were studied. Further investigation will be needed to test whether different objective functions would be involved during obstacle crossing with the trailing limb.

Furthermore, further inclusion of motion components of the frontal and transverse planes may be needed to study the motor control of obstacle crossing, especially for people with neuromusculoskeletal disorders. In the current study, both subject groups were healthy adults without any neuromusculoskeletal dysfunction and with normal or corrected vision. Further studies will be needed to identify the sensitivities of the best- 
compromise weighting sets to factors that may affect the control of obstacle crossing, such as muscle strength, proprioception, vision, spatial cognition, or fear of tumbling. Another limitation of the current study was that the cognitive functions of the subjects were not measured. The effects of neurological performance quantified by cognitive function tests (such as MMSE or STROOP) on the biomechanical phenotype in response to physical obstacles are also an interesting topic for further study.

\section{Conclusions}

The current study used multi-objective optimal control techniques with a mechanical model of the human body to study the multi-objective optimal control strategies adopted by healthy older and young adults when crossing obstacles of different heights. The older group was found to cross obstacles with significantly increased weightings to the heel- and toe-clearances, but decreased energy expenditure weightings when compared with the young group. These best-compromise weighting sets were not affected by obstacle height, suggesting that the observed strategy was one at the central nervous system level. As a result of the altered best-compromise optimal control strategies, the older group showed increased mechanical energy expenditure, leading toe-obstacle clearance, and trailing toeobstacle distance, but decreased leading heel-obstacle distance. The older people adopted a crossing strategy different from the young, emphasizing the foot-obstacle clearance to reduce the risk of tripping over the obstacle at the expense of increased energy expenditure. It appears that the MOOC strategy adopted by the older group would rely on not only the muscular strength of the lower extremities for stance stability, but also on the ability of precision end-point control for the increased toe-obstacle clearance. Therefore, apart from factors such as vision, spatial cognition, and fear of tumbling, maintaining or improving muscle strength and the ability of limb position control are critical for safe and successful obstacle crossing in the older population.

Author Contributions: Conceptualization: C.-C.K., T.-W.L. and H.-C.H.; Methodology: C.-C.K., J.-Y.W. and T.-W.L.; Formal analysis and investigation: J.-Y.W. and S.-C.C.; Writing-original draft preparation: C.-C.K., J.-Y.W. and S.-C.C.; Writing-review and editing: T.-W.L. and H.-C.H.; Funding acquisition: T.-W.L. and H.-C.H.; Resources: C.-C.K. and J.-Y.W.; Supervision: T.-W.L. All authors have read and agreed to the published version of the manuscript.

Funding: This research was financially supported by the National Science Council, Taiwan (ROC) (NSC98-2320-B-039-041).

Institutional Review Board Statement: The study was conducted following the guidelines of the Declaration of Helsinki and approved by the Institutional Review Board of China Medical University and Hospital (IRB No. DMR98-IRB-072).

Informed Consent Statement: Informed consent was obtained from all subjects involved in the current study.

Data Availability Statement: The datasets used in the current study are available from the corresponding author on reasonable request.

Conflicts of Interest: The authors declare no conflict of interest.

\section{References}

1. Tinetti, M.E.; Speechley, M.; Ginter, S.F. Risk-Factors for Falls among Elderly Persons Living in the Community. N. Engl. J. Med. 1988, 319, 1701-1707. [CrossRef] [PubMed]

2. Di Fabio, R.P.; Kurszewski, W.M.; Jorgenson, E.E.; Kunz, R.C. Footlift asymmetry during obstacle avoidance in high-risk elderly. J. Am. Geriatr. Soc. 2004, 52, 2088-2093. [CrossRef] [PubMed]

3. Novak, A.C.; Deshpande, N. Effects of aging on whole body and segmental control while obstacle crossing under impaired sensory conditions. Hum. Mov. Sci. 2014, 35, 121-130. [CrossRef] [PubMed]

4. Kim, S.G.; Hwangbo, G. The effect of obstacle gait training on the plantar pressure and contact time of elderly women. Arch. Gerontol. Geriatr. 2015, 60, 401-404. [CrossRef] [PubMed]

5. Chen, H.L.; Lu, T.W. Comparisons of the joint moments between leading and trailing limb in young adults when stepping over obstacles. Gait Posture 2006, 23, 69-77. [CrossRef] [PubMed] 
6. Overstall, P.; Exton-Smith, A.; Imms, F.; Johnson, A. Falls in the elderly related to postural imbalance. Br. Med. J. 1977, 1, 261-264. [CrossRef] [PubMed]

7. Blake, A.J.; Morgan, K.; Bendall, M.J.; Dallosso, H.; Ebrahim, S.B.; Arie, T.H.; Fentem, P.H.; Bassey, E.J. Falls by elderly people at home: Prevalence and associated factors. Age Ageing 1988, 17, 365-372. [CrossRef] [PubMed]

8. Tinetti, M.E.; Speechley, M. Prevention of falls among the elderly. N. Engl. J. Med. 1989, 320, 1055-1059.

9. Chou, L.S.; Draganich, L.F. Stepping over an obstacle increases the motions and moments of the joints of the trailing limb in young adults. J. Biomech. 1997, 30, 331-337. [CrossRef]

10. Patla, A.; Rietdyk, S. Visual control of limb trajectory over obstacles during locomotion: Effect of obstacle height and width. Gait Posture 1993, 1, 45-60. [CrossRef]

11. Chen, H.C.; Ashton-Miller, J.A.; Alexander, N.B.; Schultz, A.B. Stepping over obstacles: Gait patterns of healthy young and old adults. J. Gerontol. 1991, 46, M196-M203. [CrossRef] [PubMed]

12. Lu, T.-W.; Chen, S.-C.; Chiu, H.-C. Best-compromise between mechanical energy expenditure and foot clearance predicts leading limb motion during obstacle-crossing. Gait Posture 2012, 36, 552-556. [CrossRef] [PubMed]

13. Lu, T.W.; Chen, H.L.; Chen, S.C. Comparisons of the lower limb kinematics between young and older adults when crossing obstacles of different heights. Gait Posture 2006, 23, 471-479. [CrossRef] [PubMed]

14. Lu, T.W.; Chen, H.L.; Wang, T.M. Obstacle crossing in older adults with medial compartment knee osteoarthritis. Gait Posture 2007, 26, 553-559. [CrossRef] [PubMed]

15. Yen, H.C.; Chen, H.L.; Liu, M.W.; Liu, H.C.; Lu, T.W. Age effects on the inter-joint coordination during obstacle-crossing. J. Biomech. 2009, 42, 2501-2506. [CrossRef]

16. Austin, G.P.; Garrett, G.E.; Bohannon, R.W. Kinematic analysis of obstacle clearance during locomotion. Gait Posture 1999, 10, 109-120. [CrossRef]

17. Sparrow, W.; Shinkfield, A.J.; Chow, S.; Begg, R. Characteristics of gait in stepping over obstacles. Hum. Mov. Sci. 1996, 15, 605-622. [CrossRef]

18. McFadyen, B. Anticipatory locomotor adjustments during obstructed human walking. Neurosci. Res. Commun. 1991, 9, 37-44.

19. Wang, T.M.; Yen, H.C.; Lu, T.W.; Chen, H.L.; Chang, C.F.; Liu, Y.H.; Tsai, W.C. Bilateral knee osteoarthritis does not affect inter-joint coordination in older adults with gait deviations during obstacle-crossing. J. Biomech. 2009, 42, 2349-2356. [CrossRef]

20. Lu, T.W.; Yen, H.C.; Chen, H.L. Comparisons of the inter-joint coordination between leading and trailing limbs when crossing obstacles of different heights. Gait Posture 2008, 27, 309-315. [CrossRef]

21. Tseng, C.; Lu, T. Minimax multiobjective optimization in structural design. Int. J. Numer. Methods Eng. 1990, 30, 1213-1228. [CrossRef]

22. Chou, L.S.; Song, S.M.; Draganich, L.F. Predicting the kinematics and kinetics of gait based on the optimum trajectory of the swing limb. J. Biomech. 1995, 28, 377-385. [CrossRef]

23. Zarrugh, M. Power requirements and mechanical efficiency of treadmill walking. J. Biomech. 1981, 14, 157-165. [CrossRef]

24. Beckett, R.; Chang, K. An evaluation of the kinematics of gait by minimum energy. J. Biomech. 1968, 1, 147-159. [CrossRef]

25. Dean, G. An analysis of the energy expenditure in level and grade walking. Ergonomics 1965, 8, 31-47. [CrossRef]

26. Nubar, Y.; Contini, R. A minimal principle in biomechanics. Bull. Math. Biophys. 1961, 23, 377-391. [CrossRef]

27. Haefeli, J.; Vogeli, S.; Michel, J.; Dietz, V. Preparation and performance of obstacle steps: Interaction between brain and spinal neuronal activity. Eur. J. Neurosci. 2011, 33, 338-348. [CrossRef]

28. Maclellan, M.J.; McFadyen, B.J. Segmental control for adaptive locomotor adjustments during obstacle clearance in healthy young adults. Exp. Brain Res. 2010, 202, 307-318. [CrossRef]

29. MacLellan, M.J. Modular organization of muscle activity patterns in the leading and trailing limbs during obstacle clearance in healthy adults. Exp. Brain Res. 2017, 235, 2011-2026. [CrossRef]

30. Erdfelder, E.; Faul, F.; Buchner, A. GPOWER: A general power analysis program. Behav. Res. Methods Instrum. Comput. 1996, 28, 1-11. [CrossRef]

31. Chen, S.C.; Hsieh, H.J.; Lu, T.W.; Tseng, C.H. A method for estimating subject-specific body segment inertial parameters in human movement analysis. Gait Posture 2011, 33, 695-700. [CrossRef] [PubMed]

32. Hong, S.W.; Leu, T.H.; Wang, T.M.; Li, J.D.; Ho, W.P.; Lu, T.W. Control of body's center of mass motion relative to center of pressure during uphill walking in the elderly. Gait Posture 2015, 42, 523-528. [CrossRef] [PubMed]

33. Hsieh, H.J.; Lu, T.W.; Chen, S.C.; Chang, C.M.; Hung, C. A new device for in situ static and dynamic calibration of force platforms. Gait Posture 2011, 33, 701-705. [CrossRef] [PubMed]

34. Chen, H.-L.; Lu, T.-W.; Lin, H.-C. Three-dimensional kinematic analysis of stepping over obstacles in young subjects. Biomed. Eng. Appl. Basis Commun. 2004, 16, 157-164. [CrossRef]

35. Lu, T.-W.; O'Connor, J. A three-dimensional computer graphics-based animated model of the human locomotor system with anatomical joint constraints. J. Biomech. 1998, 1001, 116. [CrossRef]

36. Dominguez-Zamora, F.J.; Lajoie, K.; Miller, A.B.; Marigold, D.S. Age-related changes in gaze sampling strategies during obstacle navigation. Gait Posture 2020, 76, 252-258. [CrossRef]

37. Chou, L.S.; Draganich, L.F. Increasing obstacle height and decreasing toe-obstacle distance affect the joint moments of the stance limb differently when stepping over an obstacle. Gait Posture 1998, 8, 186-204. [CrossRef] 
38. Patla, A.E.; Prentice, S.; Gobbi, T. Visual Control of Obstacle Avoidance during Locomotion: Strategies in Young Children, Young and Older Adults. In Changes in Sensory-Motor Behavior in Aging; Ferandez, A.M., Teasdale, N., Eds.; Elsevier: Amsterdam, The Netherlands, 1996; pp. 257-277.

39. Huang, S.C.; Lu, T.W.; Chen, H.L.; Wang, T.M.; Chou, L.S. Age and height effects on the center of mass and center of pressure inclination angles during obstacle-crossing. Med. Eng. Phys. 2008, 30, 968-975. [CrossRef]

40. Grillner, S.; Wallen, P. Central pattern generators for locomotion, with special reference to vertebrates. Annu. Rev. Neurosci. 1985, 8, 233-261. [CrossRef]

41. McFadyen, B.J.; Prince, F. Avoidance and accommodation of surface height changes by healthy, community-dwelling, young, and elderly men. J. Gerontol. A Biol. Sci. Med. Sci. 2002, 57, B166-B174. [CrossRef]

42. Lamoureux, E.; Sparrow, W.A.; Murphy, A.; Newton, R.U. The effects of improved strength on obstacle negotiation in communityliving older adults. Gait Posture 2003, 17, 273-283. [CrossRef]

43. Lamoureux, E.L.; Sparrow, W.A.; Murphy, A.; Newton, R.U. The Relationship Between Lower Body Strength and Obstructed Gait in Community-Dwelling Older Adults. J. Am. Geriatr. Soc. 2002, 50, 468-473. [CrossRef]

44. Muir, B.C.; Haddad, J.M.; van Emmerik, R.E.A.; Rietdyk, S. Changes in the control of obstacle crossing in middle age become evident as gait task difficulty increases. Gait Posture 2019, 70, 254-259. [CrossRef]

45. Pan, H.F.; Hsu, H.C.; Chang, W.N.; Renn, J.H.; Wu, H.W. Strategies for obstacle crossing in older adults with high and low risk of falling. J. Phys. Ther. Sci. 2016, 28, 1614-1620. [CrossRef]

46. Hass, C.J.; Gregor, R.J.; Waddell, D.E.; Oliver, A.; Smith, D.W.; Fleming, R.P.; Wolf, S.L.; Hass, C.J.; Gregor, R.J.; Waddell, D.E.; et al. The influence of Tai Chi training on the center of pressure trajectory during gait initiation in older adults. Arch. Phys. Med. Rehabil. 2004, 85, 1593-1598. [CrossRef]

47. Wolf, S.L.; Barnhart, H.X.; Ellison, G.L.; Coogler, C.E. The effect of Tai Chi Quan and computerized balance training on postural stability in older subjects. Phys. Ther. 1997, 77, 371-381. [CrossRef]

48. Wolfson, L.; Whipple, R.; Derby, C.; Judge, J.; King, M.; Amerman, P.; Schmidt, J.; Smyers, D. Balance and strength training in older adults: Intervention gains and Tai Chi maintenance. J. Am. Geriatr. Soc. 1996, 44, 498-506. [CrossRef]

49. Tse, S.K.; Bailey, D.M. T'ai chi and postural control in the well elderly. Am. J. Occup. Ther. 1992, 46, 295-300. [CrossRef]

50. Watanabe, K.; Miyakawa, T. Gait Analysis during Stepping over the Different Height of Obstacles in Aged Persons. In Vestibular and Neural Front, Proceedings of the 12th International Symposium on Posture and Gait, Matsumoto, Japan, 3-7 October 1994; Taguchi, K., Igarashi, M., Mori, S., Eds.; Elsevier: Amsterdam, The Netherlands, 1994; pp. 195-198.

51. Alexander, N.B. Postural control in older adults. J. Am. Geriatr. Soc. 1994, 42, 93-108. [CrossRef]

52. Fiatarone, M.A.; Evans, W.J. 11 The etiology and reversibility of muscle dysfunction in the aged. J. Gerontol. 1993, 48, 77-83. [CrossRef] 